www.nature.com/pj

\title{
Adhesion between polyethylenes and different types of polypropylenes
}

\author{
Jie Song ${ }^{1,4}$, Anne Bringuier ${ }^{2}$, Shingo Kobayashi ${ }^{3,5}$, Adam M Baker ${ }^{1}$ and Christopher W Macosko ${ }^{1}$
}

To understand the effect of processing and co-monomer content on interfacial adhesion, we quantified adhesion levels of bilayers of a polyethylene (PE) with various polypropylenes (PPs) prepared using bilayer co-extrusion and lamination processes. We tested adhesion between a medium-density PE (MDPE) with different types of PPs, including impact-modified PP (with various amount of ethylene), isotactic PP and ethylene-propylene random copolymers. Increasing the concentration of ethylene or ethylene-propylene rubber gave rise to increased adhesion. The impact-modified PP with 20 wt\% ethylene content exhibited adhesion with MDPE almost two orders of magnitude higher compared with other PPs. Although lamination and co-extrusion processes showed good agreement in these trends with ethylene content, the operation parameters are critical for adhesion control. For lamination, ice-water cooling generated a stronger adhesion than that with air cooling. Faster cooling rates in co-extrusion also gave rise to stronger adhesion. Increasing draw down ratio and varying flow rate to put the interface near the wall resulted in stronger adhesion. Fast quenching rate and increased crystallinity induced by drawing down are believed to be the causes. Both atomic force microscopy and transmission electron microscopic images exhibited roughened interfaces for samples with strong adhesion.

Polymer Journal (2012) 44, 939-945; doi:10.1038/pj.2012.25; published online 28 March 2012

Keywords: adhesion; crystallization; extrusion; interface; polyethylene; polypropylene

\section{INTRODUCTION}

Polyethylene (PE) and isotactic polypropylene (iPP) are the first and second highest-volume thermoplastics based on worldwide consumption. ${ }^{1}$ The advent of catalysis chemistry, new applications and an expanded user base are projected to fuel the growth of these polyolefins. PE and iPP are low-cost materials providing good mechanical properties for moderate temperature applications.

Melt blending is frequently used to extend the property range of polymers. It is also an attractive approach for recycling mixed polymer waste streams. Despite the fact that PP and PE are similar, these two polymers are thermodynamically immiscible. The incompatibility of PP and PE results in blends that have extremely low fracture strain and poor toughness. For instance, when PP and PE blends are cooled from their molten state, each phase 'solidifies by chain-folding into extended crystalline lamellae sandwiched between amorphous regions composed of disorganized looping sections of polymer.' ${ }^{2}$ To improve the compatibility of these two polymers, compatibilizers are usually required. To be effective, the compatibilizer needs to concentrate at the interface, where it can reduce interfacial tension and improve adhesion.

Interfacial adhesion is not easily examined in the dispersed domain morphology of conventional melt blends. An alternative approach takes advantage of co-extrusion to fabricate a one-dimensional model of the melt blend. Although there is a considerable body of literature $^{1,3}$ on the use of interfacial coupling reactions to form covalent bonding between different phases leading to increased adhesion, there has been very little study of the adhesion between polyolefins without any chemical reactions occurring at the interface. The mechanism of adhesion at semicrystalline polyolefin interfaces and relevant parameters that affect adhesion are reported here. The adhesion between medium-density PE (MDPE) with different types of polypropylenes (PPs), including impact-modified PP (with various amount of ethylene), iPP and ethylene-propylene random copolymers, was tested. We show that the PP copolymer type, random versus impact modified, concentration of ethylene and concentration of ethylene-propylene rubber (EPR), and also processing variables, all effect adhesive bond strength.

\footnotetext{
${ }^{1}$ Department of Chemical Engineering and Materials Science, University of Minnesota, Minneapolis, MN, USA; ${ }^{2}$ Corning Cable Systems, Hickory, NC, USA and ${ }^{3}$ Department of Chemistry, University of Minnesota, Minneapolis, MN, USA

${ }^{4}$ Current address: Dow Water \& Process Solutions, Edina, 55439 MN, USA

${ }^{5}$ Current address: Department of Biochemical Engineering, Yamagata University, Yamagata, Japan

Correspondence: Professor CW Macosko, Department of Chemical Engineering and Materials Science, University of Minnesota, 421 Washington Avenue SE, Minneapolis, MN 55455, USA.

E-mail: macosko@umn.edu

Received 9 December 2011; revised 31 January 2012; accepted 2 February 2012; published online 28 March 2012
} 


\section{EXPERIMENTAL PROCEDURE}

\section{Materials}

Linear MDPE was provided by The Dow Chemical Company (Midland, MI, USA) with trade name DHDA-8864 NT. This polymer has a density of $0.932 \mathrm{~g} \mathrm{~cm}^{-3}$ and a melt index of $0.7 \mathrm{~g}$ per $10 \mathrm{~min}\left(2.16 \mathrm{~kg}\right.$ at $\left.190^{\circ} \mathrm{C}\right)$. Highdensity PE (HDPE) was also provided by The Dow Chemical Company with trade name $25455 \mathrm{~N}$, density $0.957 \mathrm{~g} \mathrm{~cm}^{-3}$ and melt index of $25 \mathrm{~g}$ per $10 \mathrm{~min}$ $\left(2.16 \mathrm{~kg}\right.$ at $\left.190^{\circ} \mathrm{C}\right)$. The propylene-ethylene random copolymer, referred as PPE in this study, was provided by INEOS (Lausanne, Switzerland) with the trade name R12C-00. This polymer has a melt flow rate of $12 \mathrm{~g}$ per $10 \mathrm{~min}(2.16 \mathrm{~kg}$ at $230{ }^{\circ} \mathrm{C}$ ). The isotactic polypropylene, referred as iPP in this study, was provided by INEOS with the trade name H03G-00. This polymer has a melt flow rate of $3.5 \mathrm{~g}$ per $10 \mathrm{~min}\left(2.16 \mathrm{~kg}\right.$ at $\left.230^{\circ} \mathrm{C}\right)$.

Impact-modified PP (20\%), with a trade name of INEOS T10G-02 has a melt flow rate of $10 \mathrm{~g}$ per $10 \mathrm{~min}\left(2.16 \mathrm{~kg}\right.$ at $\left.230^{\circ} \mathrm{C}\right)$. Impact-modified PP (9\%), with a trade name of INEOS N05U-00 has a melt flow rate of $5 \mathrm{~g}$ per $10 \mathrm{~min}\left(2.16 \mathrm{~kg}\right.$ at $\left.230^{\circ} \mathrm{C}\right)$. The molecular characteristics of polyolefins are summarized in Table 1 . In the PP $(20 \%)$, there is about $50 \%$ of the total polymer, that is, EPR. PP (20\%) contains ethylene co-monomers. In the PP (9\%), there is about $20 \%$ of the total polymer that is EPR. Ethylene and EPR concentration in Table 1 represent the percentage of ethylene segments and EPR in total in the entire polymer, respectively.

Differential-scanning calorimetry measurements were performed with TA Instruments DSC Q1000 (TA Instruments, New Castle, DE, USA). Approximately $10 \mathrm{mg}$ of dry polymers were loaded into non-hermetic aluminum pans. Differential-scanning calorimetry scans were performed at the rate of $10^{\circ} \mathrm{C} \mathrm{min}^{-1}$ from -100 to $250^{\circ} \mathrm{C}$. Melting temperature was determined from the second scanning cycle using TA Instruments Universal Analysis 2000 software.

\section{Lamination}

All the polyolefin films $(80 \mathrm{~mm} \times 7.5 \mathrm{~mm} \times 0.4 \mathrm{~mm})$ were prepared by compression molding from pellets at $210^{\circ} \mathrm{C}$ under $2 \mathrm{MPa}$ between two polytetrafluoroethylene-coated aluminum foils (Saint-Gobain Performance Plastics, Wayne, NJ, USA). All the polyolefin films were dried in vacuum oven at room temperature overnight. After drying under vacuum, MDPE or HDPE was pressed into intimate contact with various PP films, then sandwiched between two polytetrafluoroethylene-coated aluminum foils and annealed for various times at $210^{\circ} \mathrm{C}$ under $0.1 \mathrm{MPa}$ within a rectangular mold $(80 \mathrm{~mm} \times 7.5 \mathrm{~mm} \times 0.75 \mathrm{~mm})$.

Immediately after annealing, these bilayer samples were quenched by plunging into ice water or left in the air at room temperature to study the effect of cooling rate on adhesion strength. A J-type thermocouple (Omega Engineering, Inc., Stamford, CT, USA) was inserted into the center of a bilayer sample to measure the cooling rate. The edges of bilayer samples were trimmed with a razor blade. Asymmetric dual cantilever beam adhesion tests ${ }^{4,5}$ were conducted $24 \mathrm{~h}$ after lamination. At least three samples were tested for each experimental measurement, and the mean values as well as the s.d. were calculated.

\section{Co-extrusion}

Bilayer samples of MDPE with different PPs were prepared by co-extrusion at $210^{\circ} \mathrm{C}$. Gear pumps (Zenith PEP-II, Monroe, NC, USA) were used to control the flow rate to the feedblock. The MDPE and different PPs were extruded at equal and unequal flow rates by varying the speed of the gear pumps. A detailed description of the co-extrusion line can be found in the literature. ${ }^{3,6-9}$ On the basis of the continuity equation, melt velocity in the co-extrusion dies can be deduced from the chill-roll speed and film thickness. At a total flow rate of $38.4 \mathrm{~cm}^{3} \mathrm{~min}^{-1}$ as determined from gear-pump rotation rate, the residence time of the interface traveling at equal flow rates was determined to be $9-13 \mathrm{~s}$ by using the method described by Song et al. ${ }^{3}$ Upon exiting the die land, bilayer films were drawn by stainless steel rolls chilled at $4{ }^{\circ} \mathrm{C}$. The temperature of molten polymer at the nip point was measured by an infrared thermometer (Omega Engineering, Inc.). It was only $\sim 6^{\circ} \mathrm{C}$ lower than upon the die exit. Thus, the traveling time between the die exit and nip point was also included in the above residence time calculation. The die exit has dimension of $50 \mathrm{~mm} \times 1.2 \mathrm{~mm}$. The thickness of bilayer samples varied from $0.4 \mathrm{~mm}$ to $1 \mathrm{~mm}$, depending on take-up
Table 1 Molecular characteristics of polyolefins used

\begin{tabular}{lcccc}
\hline & $\begin{array}{c}\text { Ethylene } \\
\text { concentration } \\
(w t \%)\end{array}$ & $\begin{array}{c}\text { EPR } \\
\text { concentration } \\
(w t \%)\end{array}$ & $\begin{array}{c}\text { Melting } \\
\text { point } \\
\left({ }^{\circ} \mathrm{C}\right)\end{array}$ & $\begin{array}{r}\text { Heat of } \\
\text { fusion } \\
\left(\mathrm{Jg}^{-1}\right)\end{array}$ \\
\hline iPP & 0 & 0 & 162.8 & 67.9 \\
PPE & 3.4 & 0 & 148.8 & 58.7 \\
PP (9\%) & 9.1 & 20.0 & 167.4 & 72.4 \\
PP (20\%) & 19.9 & 52.0 & 146.3 & 20.5 \\
MDPE & 100 & 0 & 123.4 & 102.5 \\
HDPE & 100 & 0 & 125.0 & \\
\hline
\end{tabular}

Abbreviations: HDPE, high-density polyethylene; iPP, isotactic polypropylene; MDPE, medium-density polyethylene; PPE, propylene-ethylene random copolymer; PP, polypropylene.

velocities of the chill rolls. Samples $\sim 8 \mathrm{~mm}$ wide and $100 \mathrm{~mm}$ long were cut from the center of the co-extruded films, and asymmetric dual cantilever beam tests were conducted to test the interfacial adhesion.

\section{Characterization}

Atomic force microscopy. The cryo-microtomed cross-sections of co-extruded bilayers were examined using atomic force microscopy (AFM) with a Nanoscope III Multimode system (Digital Instruments, Santa Barbara, CA, USA) in tapping mode within the repulsive regime. All the data were acquired in height and phase mode with a scan rate of $1 \mathrm{~Hz}$.

Transmission electron microscopy. A JEOL 1200 EXII transmission electron microscope (TEM, JEOL, Peabody, MA, USA) was used to study the interface and also morphologies near the interface of cryo-microtomed cross-sections of co-extruded bilayers. An accelerating voltage of $120 \mathrm{kV}$ was used. A side-mounted, SIS MegaView III high-resolution CCD camera (Olympus Soft Imaging Solutions, Center Valley, PA, USA) and a Gatan $651 \mathrm{~N}$ liquid nitrogen anti-contaminator (Gatan Inc., Pleasanton, CA, USA) were used.

\section{RESULTS AND DISCUSSION}

\section{Lamination}

The iPP, propylene ethylene random copolymer (PPE), impactmodified PP with 9 and $20 \%$ of ethylene, that is, PP (9\%) and PP (20\%), were laminated with MDPE and HDPE by hot pressing. The adhesion strength between MDPE with various PPs was examined with two different cooling rates, air cooling $\left(\sim 5^{\circ} \mathrm{Cs}^{-1}\right)$ versus icewater cooling $\left(\sim 50^{\circ} \mathrm{Cs}^{-1}\right)$. Figures 1 and 2 show: (1) for all the polymer pairs, ice-water cooling generated stronger adhesion compared with that of air cooling; (2) PP (20\%) gave rise to adhesion that was two orders of magnitude higher than the rest of the PP materials. We examine these two findings in the following paragraphs.

Narasimhan and colleagues ${ }^{10}$ conducted elegant experiments to determine how temperature change will affect the interfacial width between immiscible polymers. They found that when iPP and PE were annealed at $160^{\circ} \mathrm{C}$, the equilibrium interfacial width was approximately $28 \mathrm{~nm}$. When the annealing temperature was changed to $140{ }^{\circ} \mathrm{C}$, the equilibrium interfacial width decreased to $11 \mathrm{~nm}$. If the laminates were annealed at $160^{\circ} \mathrm{C}$, then quenched to $140^{\circ} \mathrm{C}$, the interfacial width was approximately $21 \mathrm{~nm}$, between the values observed in the two simple annealing experiments.

The ethylene-propylene segmental interaction parameter $\chi$ follows an inverse dependence on temperature ${ }^{10}$

$$
\chi=A+\frac{B}{T}
$$

where $A$ is a negative constant and $B$ is a positive constant. Apparently, lower temperature leads to larger $\chi$, indicating poor 


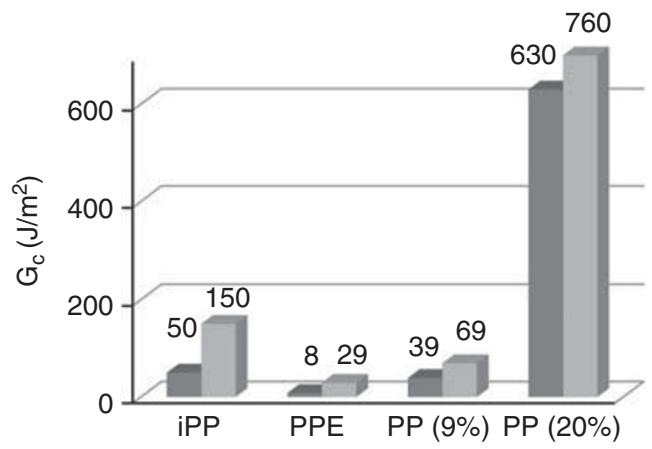

Figure 1 Adhesion between MDPE with various PPs under different cooling conditions. For each PP, the left column represents air cooling; the right column represents ice-water cooling. A full color version of this figure is available at Polymer Journal online.

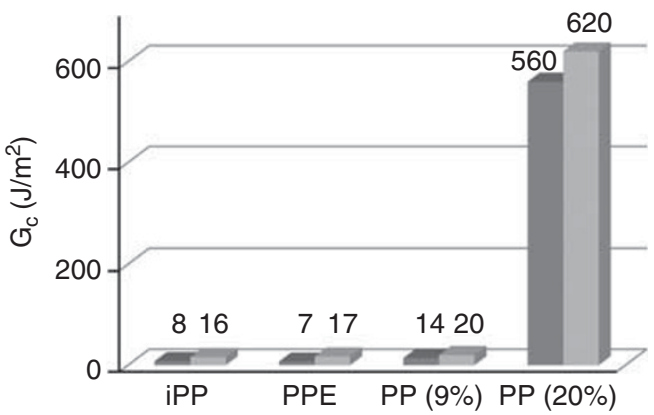

Figure 2 Adhesion between HDPE with various PPs under different cooling conditions. For each PP, the left column represents air cooling; the right column represents ice-water cooling. A full color version of this figure is available at Polymer Journal online.

compatibility. From Narasimhan's findings and the dependence of $\chi$ on temperature, we can tell that although lower annealing temperature generates thinner interface due to larger $\chi$, quenching can help to retain the entangled structure generated from high temperature.

As the interface cools, the interface tends to shrink, because iPP and PE become more immiscible. Fast cooling and fast crystallization freeze the interface before it has time to shrink. Interfacial entanglements established in the molten state are anchored in chain-folded lamellae upon quenching. Slower cooling provides polymer chains more time to diffuse out of the interfacial region and come closer to the thin interfacial structure at low temperature before freezing. Therefore, faster cooling rate facilitates preservation of the entanglement structure created at high temperature. In contrast, slower cooling helps the interface to reach equilibrium state at low temperature with less entanglement and thinner interface.

Regarding the dramatic adhesion improvement by using PP (20\%), we propose that interfacial adhesion is dominated by compatibility. Increasing the concentration of ethylene raises the compatibility of PP (20\%) with MDPE and HDPE. Zhu et al. ${ }^{11}$ also observed that increasing the ethylene content of an ethylene-propylene copolymer at the $\mathrm{PE} / \mathrm{iPP}$ interface enhanced mechanical properties of these blends, indicating stronger interfacial bonding. Therefore the cocrystallization of the ethylene segments in the ethylene-propylene copolymer component of PP (20\%), and the PE chain in HDPE was proposed to explain the high-strength welding. This could also be due to more entanglement, although it is difficult to differentiate cocrystallization from entanglement with the current data. It should be
Table 2 Adhesion strength of co-extruded bilayers with different processing variables

\begin{tabular}{|c|c|c|c|c|c|c|}
\hline Sample & Material 1 & Material 2 & $Q_{1} / Q_{2}$ & $\begin{array}{c}Q_{\text {total }} \\
\left(\mathrm{cm}^{3} \mathrm{~min}^{-1}\right)\end{array}$ & $\begin{array}{c}\text { Take-up } \\
\text { speed } \\
\left(\mathrm{cm} \mathrm{min}^{-1}\right)\end{array}$ & $\begin{array}{c}\text { Adhesion } \\
\text { strength } \\
\left(\mathrm{J} \mathrm{m}^{-2}\right)\end{array}$ \\
\hline 1 & MDPE & iPP & $1 / 1$ & 33.6 & 64.7 & $55 \pm 12$ \\
\hline 2 & MDPE & iPP & $1 / 1$ & 33.6 & 108.3 & $77 \pm 21$ \\
\hline 3 & MDPE & iPP & $1 / 1$ & 33.6 & 146.4 & $251 \pm 67$ \\
\hline 4 & MDPE & iPP & $1 / 6.25$ & 34.8 & 64.7 & $588 \pm 108$ \\
\hline 5 & MDPE & PPE & $6.25 / 1$ & 34.8 & 64.7 & $279 \pm 47$ \\
\hline 6 & MDPE & PPE & $6.25 / 1$ & 34.8 & 108.3 & $187 \pm 39$ \\
\hline 7 & MDPE & PPE & $6.25 / 1$ & 34.8 & 146.4 & $152 \pm 45$ \\
\hline 8 & MDPE & PPE & $6.25 / 1$ & 34.8 & 185.2 & $120 \pm 25$ \\
\hline 9 & MDPE & PPE & $1 / 1$ & 33.6 & 64.7 & $79 \pm 21$ \\
\hline 10 & MDPE & PPE & $1 / 1$ & 33.6 & 108.3 & $62 \pm 16$ \\
\hline 11 & MDPE & PPE & $1 / 1$ & 33.6 & 146.4 & $54 \pm 14$ \\
\hline 12 & MDPE & PPE & $1 / 1$ & 33.6 & 185.2 & $42 \pm 12$ \\
\hline 13 & MDPE & PP (9\%) & $12.5 / 1$ & 32.4 & 64.7 & $224 \pm 38$ \\
\hline 14 & MDPE & PP (9\%) & $12.5 / 1$ & 32.4 & 108.3 & $294 \pm 58$ \\
\hline 15 & MDPE & PP (9\%) & $12.5 / 1$ & 32.4 & 146.4 & $485 \pm 75$ \\
\hline 16 & MDPE & PP (20\%) & $1 / 1$ & 33.6 & 64.7 & $1437 \pm 308$ \\
\hline 17 & MDPE & PP (20\%) & $1 / 1$ & 33.6 & 108.3 & $1976 \pm 462$ \\
\hline 18 & MDPE & PP (20\%) & $1 / 1$ & 33.6 & 146.4 & $2310 \pm 508$ \\
\hline 19 & MDPE & PP (20\%) & $6.25 / 1$ & 34.8 & 64.7 & $1524 \pm 377$ \\
\hline 20 & MDPE & PP (20\%) & $1 / 6.25$ & 34.8 & 64.7 & $1918 \pm 515$ \\
\hline
\end{tabular}

Abbreviations: HDPE, high-density polyethylene; iPP, isotactic polypropylene; MDPE, medium density polyethylene; PPE, propylene-ethylene random copolymer; PP, polypropylene.

noted that MDPE showed stronger adhesion with each one of the PPs compared with HDPE. We attribute this to the better compatibility of MDPE than HDPE with PPs.

\section{Co-extrusion}

The flow-rate ratio, total flow rate, take-up speed and adhesion strength are summarized in Table 2 . We intentionally controlled the total flow rate to be almost identical, so that the residence time of the bilayers will not contribute to the change of adhesion. We varied the flow-rate ratio to apply different levels of shear stress to the interface for the same pair of materials. When the flow rate of one layer is identical to that of the other layer, that is, with an equal proportion of $\mathrm{PE}$ and PP, the interface between the two materials within the extrusion tooling is located at the center of the flow and thus experiences zero shear stress. However, when the ratio between the PP and MDPE thickness is changed, the interface between the two materials within the tooling moves to a region of non-zero shear stress.

We found that for the same polymer pair, unequal flow rate exhibit stronger adhesion compared with equal flow rate. For instance, as shown in Table 2, co-extrusion sample 5-8 generally demonstrated stronger adhesion compared with sample 9-12, whereas all the other parameters are the same. Similarly, sample 4 exhibited stronger adhesion than sample 1 , and 19 and 20 are stronger than 16. As discussed above, with unequal flow rates, the interface between the two materials within the tooling moves to a region of non-zero shear stress. We have shown that shear has a role of disentangling the interface and lowering the adhesion. ${ }^{12}$ However, we estimate the shear stress even for the $12.5 / 1$ flow ratios to be $13.3 \mathrm{KPa}$, significantly less 
than stresses needed to reduce adhesion. ${ }^{6,7}$ Therefore, we attribute this adhesion difference to different cooling rates at the interface.

To estimate the cooling rates at the interface when the bilayer went through the nip point of the chill rolls, the unsteady state heat conduction model was used as described by Bird et al. ${ }^{13}$

$$
\frac{T_{\mathrm{b}}-T}{T_{\mathrm{b}}-T_{0}}=2 \sum \frac{(-1)^{n}}{\left(n+\frac{1}{2}\right) \pi} e^{-\left(n+\frac{1}{2}\right)^{2} \pi^{2} \frac{\alpha \mathrm{t}}{l^{2}}} \cos \left(n+\frac{1}{2}\right) \frac{\pi \mathrm{z}}{l_{0}}
$$

where $T_{\mathrm{b}}$ is temperature of chill roll, $T_{0}$ is temperature of the polymer melt at time zero, $T$ is temperature of position $\mathrm{z}$ at time $\mathrm{t}, \alpha$ is thermal diffusivity, $l_{0}$ is half of the gap between the two rolls, and $\mathrm{z}$ is distance from the center of the polymer melt to surface. It was determined that the cooling rate at the interface of sample 5 is almost five times of that of sample 9 due to thickness difference. As shown in the lamination tests, faster cooling generated stronger adhesion between the immiscible polyolefins.

As the residence time for the interface traveling at unequal flow rates is greater than that at equal flow rates, one could argue that the longer residence time would lead to more time for entanglement at the interface and thus higher adhesion. However, we determined that the reptation time is much smaller than the residence time as shown below; thus, no matter where the interface is located, it would have plenty of time for entanglements to build up. Therefore, there must be some other cause for the difference of adhesion.

At a total flow rate of $38.4 \mathrm{~cm}^{3} \mathrm{~min}^{-1}$, the residence time of the interface traveling at equal flow rates was determined to be 9 to $13 \mathrm{~s}$ by using the method described by Song et al. ${ }^{3}$ From the cross-over frequency of $\mathrm{G}^{\prime}$ and $\mathrm{G}^{\prime \prime}$ at $210^{\circ} \mathrm{C}$, the longest relaxation time for PP (9\%) was determined to be $0.025 \mathrm{~s}$. Obviously, it is much shorter than the residence time at equal flow rate. Considering the reptation time is even smaller than the longest relaxation time, the reptation time of this polymer is much smaller than the residence time at equal flow rates. As the residence time at unequal flow rate is longer than that at equal flow rate, the reptation time is also smaller than the residence time at unequal flow rates. The same argument holds for other polymers too.

\section{Morphologies near interface and interface characterization}

$\chi$ has an important role in terms of determining the interfacial width and adhesion. ${ }^{14}$ Chaturvedi et al. ${ }^{15}$ used the mean-field theory to propose a $\chi$-dependent interfacial width $(w)$ for incompatible polymers:

$$
w=\frac{b \sqrt{2}}{3 \chi_{c}^{0.5}}\left(\frac{\chi}{\chi_{c}}-1\right)^{-1 / 2}
$$

where $b$ is the average segment length and $\chi_{c}$ is the interaction parameter at the critical point. $\chi_{c}$ can be obtained by the equation from Krause et al.: ${ }^{16}$

$$
\chi_{c}=\frac{1}{2}\left[x_{1}^{0.5}+x_{2}^{0.5}\right]^{2}
$$

where the $x_{\mathrm{i}}$ is the degree of polymerization of the polymers. For our system, $\chi_{c}$ was estimated to be $2 \times 10^{-3}$ by plugging in the molecular weight into equation (4).

The average segment length $b$ can be calculated as follows:

$$
b=\left(\frac{b_{i P P}^{2}+b_{P E}^{2}}{2}\right)^{1 / 2}
$$

The segment lengths of PE and iPP are 2.55 and $6.5 \AA$, respectively. ${ }^{17}$ Therefore, the average segment length $b$ was determined to be $4.93 \AA$. By using the $\chi$-value determined by Narasimhan et al. ${ }^{10}$ from the SAXS data, we were able to calculate the interfacial width. Table 3 compares the interfacial width obtained from TEM with the value
Table 3 Comparison of the interfacial widths obtained from TEM and calculated with equation (1)

\begin{tabular}{lcc}
\hline Sample & $\begin{array}{c}\text { Interfacial width obtained } \\
\text { from TEM (nm) }\end{array}$ & $\begin{array}{c}\text { Interfacial width calculated } \\
\text { from equation (3) (nm) }\end{array}$ \\
\hline 13 & 20.6 & 10.9 \\
15 & 48.5 & 10.9 \\
\hline
\end{tabular}

Abbreviation: TEM, transmission electron microscopy.
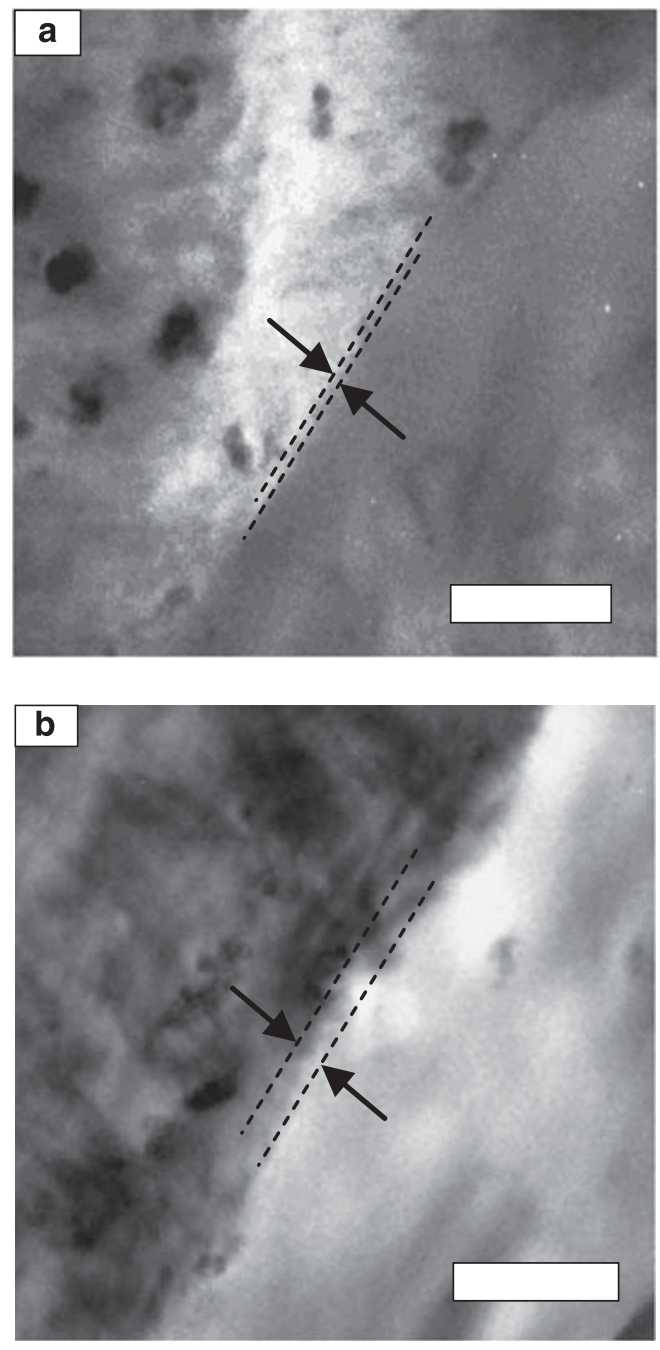

Figure 3 (a) Interfacial morphology as observed with TEM for co-extrusion sample 13. The top left phase is MDPE with carbon black; the bottom right phase is PP (9\%). (b) Interfacial morphology as observed with TEM for coextrusion sample 15 . The top left phase is MDPE with carbon black; the bottom right phase is PP (9\%). The dotted lines mark the approximate interfacial width. Scale bars represent $500 \mathrm{~nm}$.

calculated with equation (3). The observed interfacial widths, as shown in Figure 3, are significantly wider than the calculated ones. Calculations assume equilibrium while co-extrusion samples were rapidly quenched. Sample 15 was quenched about four times faster than sample 13, and its interfacial width is almost twice that of coextrusion sample 13. As discussed previously in the lamination session, the fast quenching induced by higher take-up speed helps 


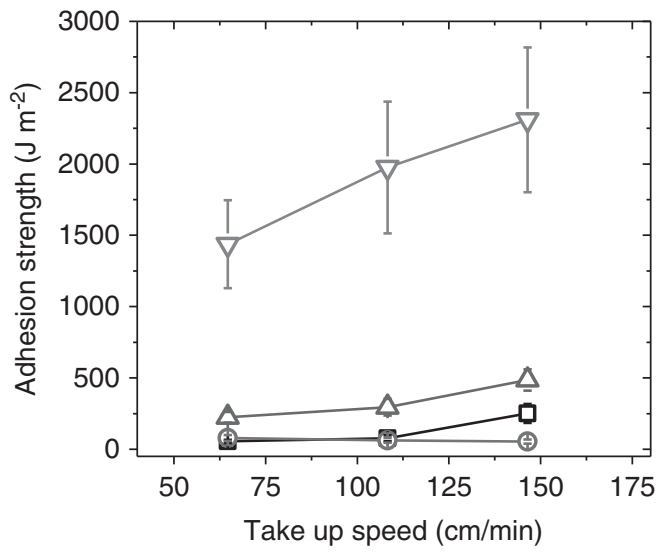

Figure 4 Variation of adhesion strength as a function of take-up speed during co-extrusion. All the data in the plot are taken from Table 2. $\nabla$ : PP (20\%) Sample 16-18; $\Delta$ : PP (9\%) Sample 13-15; $\square$ : iPP Sample 1-3; O: PPE Sample 9-12. A full color version of this figure is available at Polymer Journal online.

Table 4 The effect of take-up speed on adhesion correlated with crystallinity

\begin{tabular}{lccc}
\hline Sample & $\begin{array}{c}\text { Take-up speed } \\
(\mathrm{cm} \mathrm{min}-1)\end{array}$ & $\begin{array}{c}\text { Adhesion strength } \\
\left(\mathrm{J} \mathrm{m}^{-2}\right)\end{array}$ & $\begin{array}{c}\text { Crystallinity of } \\
\text { iPP (\%) }\end{array}$ \\
\hline 1 & 64.7 & 55 & 33.5 \\
2 & 108.3 & 77 & 40.9 \\
3 & 146.4 & 251 & 43.4 \\
\hline
\end{tabular}

Abbreviation: iPP, isotactic polypropylene

retain the more compatible structure at high temperature, where the interface should be broader.

Table 2 also showed that increasing take-up speed leads to stronger interfacial bonding. Figure 4 shows that from co-extrusion sample 1 to 3 , adhesion strength increased by almost five times when the takeup speed increased. PP (20\%) and PP (9\%) also showed similar trend. Note that before the bilayer streams exited the die, the flow conditions were all the same for sample 1 to 3 . The difference was that, in addition to faster cooling, upon exiting the die polymer chains were extended to different degrees. Increasing take-up speed resulted in increased crystallinity, as shown in Table 4. It is not surprising that increased crystallinity leads to stronger interfacial adhesion, as proved by other researchers as well. ${ }^{4,5}$ Faster take-up speeds also lead to smaller thickness, giving rise to faster cooling rate as equation (2) suggested. Faster cooling rate will also result in stronger adhesion as discussed above.

Figure 5 gave us more insights to the microstructure of the impactmodified PP (9\%) polymer. The AFM test was conducted in the repulsive regime; the dark phase indicates softer regions, whereas the brighter phase represents harder ones. The dark inclusions (soft) with hard inclusions in them, probably crystallizable EPR, are a classic signature for Ziegler Natta iPP with some EPR in it.

Figure 6 showed that near the interface of iPP/MDPE sample 4 Figure $6 \mathrm{~b}$, there are many small crystals in contrast to sample 1 Figure 6a. This is due to the fact that the interface of sample 4 is closer to the surface and cools much faster. It has a more roughened interface compared with sample 1 that has a clear, sharp interface. It is


Figure 5 Morphology of cross-section of co-extruded bilayers. (a) MDPE versus PP $(9 \%)$ with flow-rate ratio 1:1 (b) Magnified image of (a). The left phase is PP (9\%) and the right is MDPE. The arrows indicate interface. The scale bar in (a) represents $5 \mu \mathrm{m}$. The scale bar in (b) represents $1 \mu \mathrm{m}$.

not surprising that sample 4 with more roughened interface has much stronger adhesion compared with sample 1 with a sharp interface. There are numerous examples in literature ${ }^{18,19}$ showing that strong interfacial adhesion between immiscible polymers usually results from roughened interfaces.

Figure 7 showed the morphologies of PP (20\%) near the interface. For the image Figure $7 \mathrm{a}$ in which the interfacial shear stress was more than zero, the EPR rubber domains were distorted and ruptured, whereas for the image Figure $7 \mathrm{~b}$ in which the interfacial shear stress was zero, the EPR domains maintained their droplet structure. Both images also displayed significant EPR in contact with the MDPE phase providing the interfacial adhesion, whereas for PP (9\%) as shown in Figure 5, almost no EPR was contacting the MDPE phase. Figures 1 and 2 and Table 2 show that PP (20\%) exhibits dramatic 

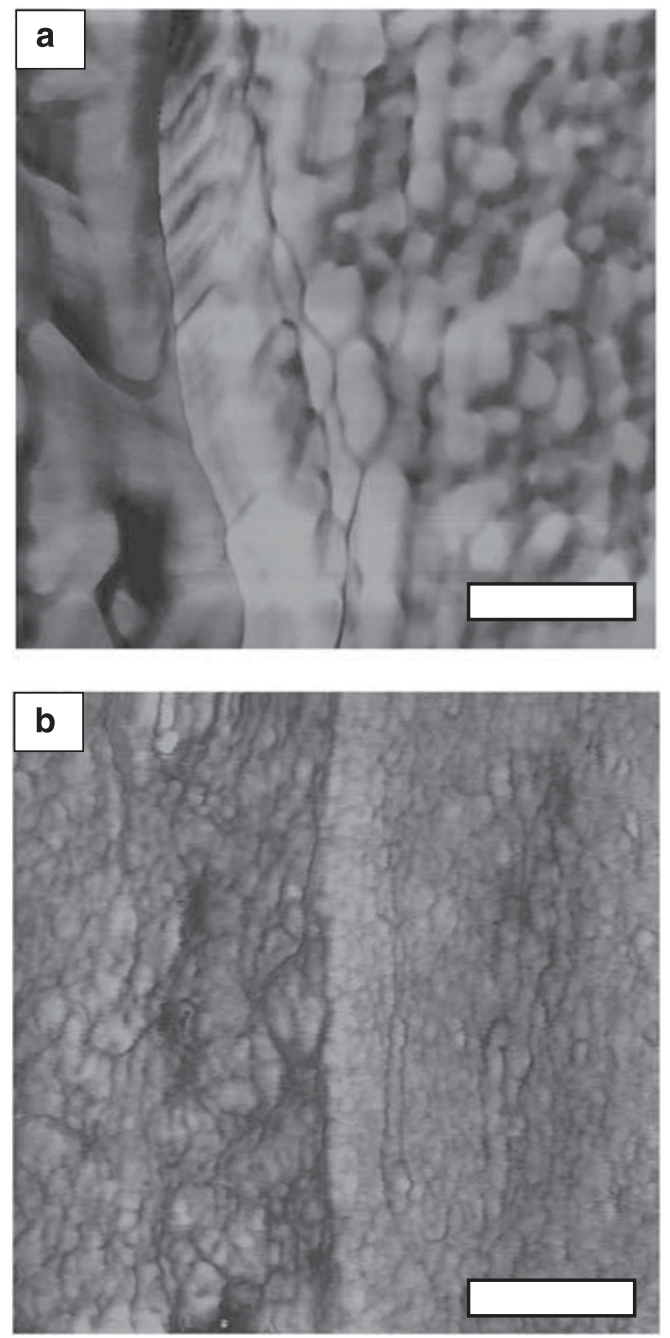

Figure 6 Morphology of cross-section of co-extruded bilayers. (a) Coextrusion sample 1. (b) Co-extrusion sample 4. For both images, the left side is MDPE and the right is iPP. Faster cooling for sample 4 generated smaller crystalline domains than sample 1 . The scale bar represents $250 \mathrm{~nm}$. A full color version of this figure is available at Polymer Journal online.

adhesion improvement with MDPE and HDPE. Therefore, the EPR showed remarkable compatibility with MDPE and HDPE. Considering the fact that there was more than $50 \%$ of ethylene in the EPR phase, clearly the large amount of ethylene gave rise to the much better compatibility with the PE phase; hence, the strong interfacial adhesion.

\section{CONCLUSION}

Adhesion between PE and various PP were investigated both through lamination and co-extrusion processes; both showed similar results. Cooling rate is critical in terms of determining interfacial adhesion between polyolefins. For all the laminated polymer pairs, ice-water cooling generated stronger adhesion compared with that with air cooling. Faster cooling rates in co-extrusion also gave rise to stronger adhesion and more roughened interfaces, as verified by utilizing unequal flow rates, which places the interface closer to the chill-roll surface. By simply increasing the take-up speed of the chill rolls, we increased interface adhesion strength at least by a factor of 2. Faster
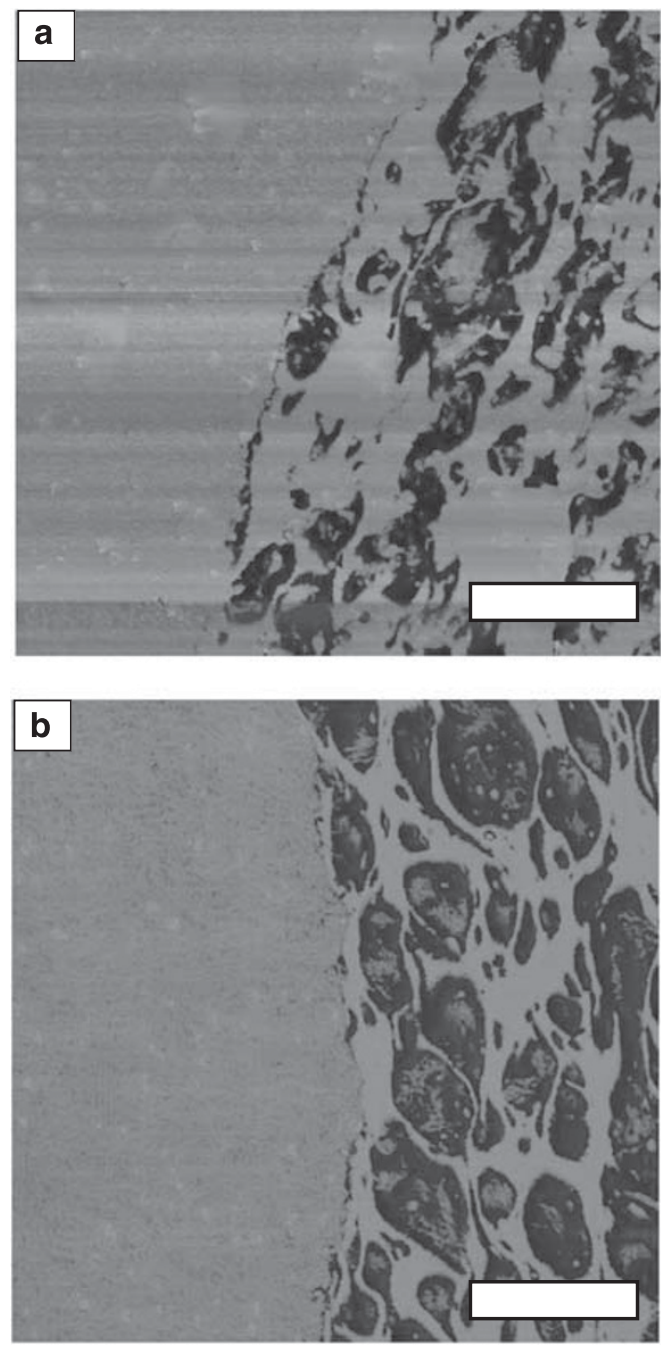

Figure 7 Morphology of cross-section of co-extruded bilayers. (a) Coextrusion sample 19. (b) Co-extrusion sample 16. The left side is MDPE and the right is $\mathrm{PP}(20 \%)$. The scale bar represents $2.5 \mu \mathrm{m}$. A full color version of this figure is available at Polymer Journal online.

cooling and increased crystallinity induced by drawing down of the chill rolls is believed to cause stronger adhesion. Material composition, specifically the percentage of ethylene, determines the compatibility of the PP materials with PE; hence, the interfacial adhesion. Impact-modified PP with $20 \%$ of ethylene gave rise to adhesion almost two orders of magnitude higher than the other PP materials. Both AFM and TEM characterization proved to be valuable techniques to reveal the interfacial structure and morphologies of the polyolefins near the interface. AFM and TEM images exhibited roughened interfaces for samples with stronger adhesion.

\section{ACKNOWLEDGEMENTS}

This research was supported by Corning Cable through their membership in the University of Minnesota Industrial Partnership for Research in Interfacial and Materials Engineering (IPRIME) and Anne Bringuier's fellowship under this partnership. We also appreciate valuable help from Greg Haugstad with AFM and Fang Zhou with TEM, both from the College of Science and Engineering Characterization Facility, supported in part by grants from NSF through the MRSEC and NNIN programs. 
1 Kobayashi, S., Song, J., Silvis, H. C., Macosko, C. W. \& Hillmyer, M. A. Aminofunctionalized polyethylene for enhancing the adhesion between polyolefins and polyurethanes. Ind. Eng. Chem. Res. 50, 3274-3279 (2011).

2 Chaffin, K. A., Knutsen, J. S., Brant, P. \& Bates, F. S. High-strength welds in metallocene polypropylene/polyethylene laminates. Science 288, 2187-2190 (2000).

3 Song, J., Ewoldt, R. H., Hu, W., Silvis, H. C. \& Macosko, C. W. Flow accelerates adhesion between functional polyethylene and polyurethane. AlChE J 57, 3496-3506 (2011).

4 Boucher, E., Folkers, J. P., Hervet, H., Leger, L. \& Creton, C. Effects of the formation of copolymer on the interfacial adhesion between semicrystalline polymers. Macromolecules 29, 774-782 (1996).

5 Boucher, E., Folkers, J. P., Creton, C., Hervet, H. \& Leger, L. Enhanced adhesion between polypropylene and polyamide- 6 : role of interfacial nucleation of the betacrystalline form of polypropylene. Macromolecules 30, 2102-2109 (1997).

6 Zhang, J., Ji, S., Song, J., Lodge, T. P. \& Macosko, C. W. Flow accelerates interfacial coupling reactions. Macromolecules 43, 7617-7624 (2010).

7 Zhao, R. \& Macosko, C. W. Slip at polymer-polymer interfaces: rheological measurements on coextruded multilayers. J. Rheol. 46, 145-167 (2002).

8 Lee, P. C., Park, H. E., Morse, D. C. \& Macosko, C. W. Polymer-polymer interfacial slip in multilayered films. J. Rheol. 53, 893-915 (2009).

9 Dolgovskij, M. Dispersing layered silicates in polymer melts: using melt rheology to determine dispersion. PhD Thesis (University of Minnesota, 2007).
10 Lo, C. T., Laabs, F. C. \& Narasimhan, B. Interfacial adhesion mechanisms in incompatible semicrystalline polymer systems. J. Polym. Sci.: Polym. Phys 42, 2667-2679 (2004).

11 Zhu, W., Zhang, X. Q., Huang, B. T. \& Feng, Z. L. Modification of PP/HDPE blends by PP-PE sequential polymerization product. J. Appl. Polym. Sci. 58, 515-521 (1995).

12 Zhang, J., Lodge, T. P. \& Macosko, C. W. Interfacial slip reduces polymer-polymer adhesion during coextrusion. J. Rheol. 50, 41-57 (2006).

13 Bird, R. B., Stewart, W. E. \& Lightfoot, E. N. Transport Phenomena (John Wiley \& Sons, New York (2007)).

14 Jablonski, E. L., Gorga, R. E. \& Narasimhan, B. Interdiffusion and phase behavior at homopolymer/random copolymer interfaces. Polymer 44, 729-741 (2003).

15 Chaturvedi, U. K., Steiner, U., Zak, O., Krausch, G. \& Klein, J. Interfacial structure in polymer mixtures below the critical-point. Phys. Rev. Lett. 63, 616-619 (1989).

16 McEvoy, R. L. \& Krause, S. Interfacial interactions between polyethylene and polypropylene and some ethylene-containing copolymers. Macromolecules 29, 4258-4266 (1996).

17 Lin, Y. J., Poon, B. C., Marchand, G. R., Hiltner, A. \& Baer, E. Adhesion of propyleneethylene copolymers to high-density polyethylene. Polym. Eng. Sci. 50, 592-605 (2010).

18 Lyu, S. P., Cernohous, J. J., Bates, F. S. \& Macosko, C. W. Interfacial reaction induced roughening in polymer blends. Macromolecules 32, 106-110 (1999).

19 Potschke, P., Pionteck, J. \& Stutz, H. Surface tension, interfacial tension, and morphology in blends of thermoplastic polyurethanes and polyolefins. Part I. surface tension of melts of Tpu model substances and polyolefins. Polymer 43, 6965-6972 (2002). 